\title{
Conceptual Study on Flexible Guidance and Navigation for ITER Remote Handling Transport Casks*
}

\author{
M. Isabel Ribeiro, Pedro Lima, Pedro Aparício, Renato Ferreira \\ Instituto Superior Técnico/ Instituto de Sistemas e Robótica, \\ Av.Rovisco Pais 1, P-1096 Lisboa CODEX, PORTUGAL \\ Tel: 35118418059 , Fax: 35118418291 , email: mir@isr.ist.utl.pt
}

\begin{abstract}
This paper presents a conceptual study on flexible guidance and navigation solutions for a remote transport system to be used in the International Thermonuclear Experimental Reactor (ITER), as opposed to hardguidance solutions such as rails. The kinematic structure of the transporters and guidance and navigation strategies are the main addressed issues.
\end{abstract}

\section{INTRODUCTION}

This paper presents a conceptual study on flexible guidance and navigation solutions for the remote transport system of the International Thermonuclear Experimental Reactor (ITER). The transport vehicles under study will operate between the Vacuum-Vessel (VV) and the HotCell Building (HCB) and must be capable of safely moving 20-80 tons of radioactive materials, their major task being the secure transfer of active in-vessel components. The path to be followed by the transportation vehicles includes line segments and curves of different radius, notably $37 \mathrm{~m}$ in the gallery and $5 \mathrm{~m}$ to change from the gallery circular path to a radial path towards the docking port, and requires interfacing with the $\mathrm{VV}$ docking ports, a lift, and the HCB docking ports.

Vehicles may have different structures regarding traction and steering. Different structures mean different maneuvering capabilities, different mechanical designs and different spanned areas when turning. Three kinematic structures are presented in Section II along with some discussion on the use of air cushion principles for the replacement of free wheels. Different types of guidance and navigation concepts are discussed in Section III.

The study focus on the advantages, disadvantages and requirements of flexible guidance and navigation solutions, as compared to "hard-guidance" solutions such as rails, in the specific ITER application described above. Section IV points out towards a flexible solution for the referred problem.

\section{Kinematics of Platforms}

Non hard guidance solutions for the transport vehicles may present different degrees of flexibility, ranging from the classical AGVs, that follow a path physically defined at the floor level, to the mobile robots that may freely move in all empty space. In between, there are the mixedtype Vehicles with the capability to switch between AGVlike and mobile Robot -like behaviours.

\footnotetext{
* This work was done under the contract ERB 5004 CT 960127 NET/96-341 between the European Atomic Energy Community and the Association EURATOM/IST.
}

In this section, different kinematic structures are studied. The spanned areas when turning, the minimum turning radius and the need for maneuvers are analyzed. Any of the kinematic structures apply to the three different types of vehicles above referred.

\section{A. Kinematics}

The trajectory topology strongly depend on the kinematic structure of the transport vehicles. The structures analyzed applies to AGVs, mobile robots and to mixed-t ype vehicles. The kinematics of the vehicles depends on the type of traction and steering used. Three different structures are analyzed here: cart like vehicles (similar to tricycle), rombic configuration with differential steering and rombic configuration with one drive and steering wheel plus a steering wheel.

\section{Cart}

Cart like vehicles are similar to cars regarding its traction and steering systems as displayed in Figure 1 where the drive and steering wheels are shaded. To carry heavy loads, more wheels are needed (as in trucks) bringing problems to the steering system (some wheels will slide sideways). To overcome this problem, it is possible to steer all the wheels but this leads to a much more complex steering system. These vehicles are not well suited to be bidirectional (move forward and backward) because of its lack of symmetry.

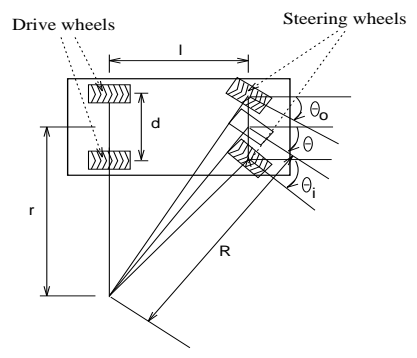

Fig. 1. Cart like vehicle and associated variables

Cart like vehicles are equivalent to tricycle vehicles when the relation between the steering angles of both wheels (Figure 1 ) is given by $\cot \theta=\cot \theta_{i}+\frac{d}{2 l}=\cot \theta_{0}-\frac{d}{2 l}$. The curvature radii relative to the mid point between the fixed rear wheels and an equivalent front steering wheel (see Figure 1) are given by $R=\frac{l}{\sin \theta}$ and $r=$ $\frac{l}{\tan \theta}$. Clearly there is a minimum turning radius equal to $r_{\min }=\frac{l}{\tan \theta_{\max }}$. Table I presents some radii of curvature for different steering angles and distance between axis. 
Table I

Curvature radii of cart vehicle

\begin{tabular}{|c|c|c|c|}
\hline $\begin{array}{c}\text { Steering } \\
\left.\text { angle }(\theta){ }^{\circ}\right]\end{array}$ & $\begin{array}{c}\text { Distance } \\
\text { btw. axis }(l)[\mathrm{m}]\end{array}$ & $\begin{array}{c}\text { Curvature } \\
\text { radius }(r)[\mathrm{m}]\end{array}$ & $\begin{array}{c}\text { Curvature } \\
\text { radius }(R)[\mathrm{m}]\end{array}$ \\
\hline 45 & 7.0 & 7.00 & 9.90 \\
\hline 45 & 6.0 & 6.00 & 8.49 \\
\hline 30 & 7.0 & 12.12 & 14.00 \\
\hline 30 & 6.0 & 10.39 & 12.00 \\
\hline
\end{tabular}

\section{Differential steering}

Vehicles with differential steering are based on two independent drive wheels (see Figure 2) with steering achieved by applying different velocities to each drive wheel. Drive wheels' axles must be aligned with each other and must be placed at the center of the vehicle in order to decrease spanned areas. With this structure, casters are needed to support the vehicle.

This type of vehicles has the capability to move forward and backward. Their structure is not well suited for long vehicles due to the long distance between the corners and the center of rotation (mid point of the axis between the the two drive wheels) meaning that large areas will be spanned when changing direction (e.g., switch from radial path to a circular path). Differential steering vehicles may rotate over its center of rotation. However, this movement is not advisable because of the strains caused to vehicle structure and drive wheels. For this type of vehicle, the

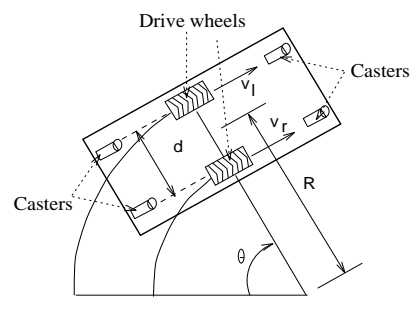

Fig. 2. Differential steering and associated variables

curvature radius of a trajectory is related with the linear velocities of both wheels by

$$
R=\frac{\left(1+\frac{v_{r}}{v_{l}}\right) \frac{d}{2}}{1-\frac{v_{r}}{v_{l}}}
$$

where $v_{r}$ and $v_{l}$ are the velocities of the left and right wheels respectively, and $d$ is the distance between wheels. There is no minimum radius of curvature constrain for these vehicles as they can rotate over themselves.

Table II presents some radii of curvature for different velocities of the wheels and distances between them. In the table, infinity $(\infty)$ radius of curvature means straight line trajectory and 0 meters means rotation over itself. Notice that a $5 \mathrm{~m}$ curvature radius is required to change from a circular path on a gallery of the VV building to a radial path towards a docking port.

\section{Rombic}

The third type of vehicle has a rombic configuration with
Table II

Curvature radii of differential drive vehicle

\begin{tabular}{|c|c|c|c|}
\hline$v_{T}[\mathrm{~cm} / \mathrm{s}]$ & $v_{l}[\mathrm{~cm} / \mathrm{s}]$ & $\begin{array}{c}\text { Distance } \\
\text { wheels }(d)[\mathrm{m}]\end{array}$ & $\begin{array}{c}\text { Curvature } \\
\text { radius }(R)[\mathrm{m}]\end{array}$ \\
\hline 28 & 28 & Any & $\infty$ \\
\hline 28 & -28 & Any & 0 \\
\hline 28 & 0 & 4 & 2.00 \\
\hline 28 & 0 & 2.5 & 1.25 \\
\hline 28 & 14 & 4 & 6.00 \\
\hline 28 & 14 & 2.5 & 3.75 \\
\hline
\end{tabular}

two independent steering wheels placed one in the front and the other in the back of the vehicle (Figure 3). One or both of these wheels is also the drive wheel. The vehicles also have free wheels or casters to support and stabilize them. By using front and rear track sensors, these vehicles are well suited for track following. They have the capability to change direction in limited spaces and thus may change between radial and circular paths. Because of its symmetry, this type of vehicle is capable to move forward and backward. The relations between steering

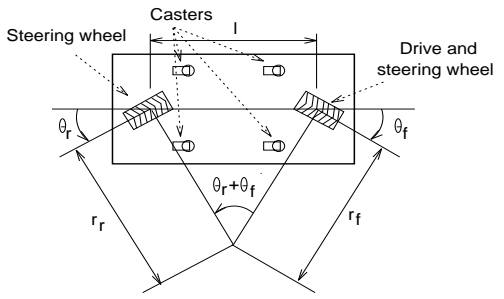

Fig. 3. Rombic vehicle and associated variables

angles of each wheel and radius of curvature are as follows:

$$
r_{f} \sin \theta_{f}+r_{r} \sin \theta_{r}=l, \quad r_{f} \cos \theta_{f}=r_{r} \cos \theta_{r}
$$

where $\mathrm{f}(\mathrm{r})$ denotes front(rear) steering wheel. If $\theta_{r}=\theta_{f}=$ $\theta$, curvature radius $\left(r=r_{r}=r_{f}\right)$ is given by $r=\frac{l}{2 \sin \theta}$ with the minimum radius of curvature given by $r_{\min }=$ $\frac{l}{2 \sin \theta_{\max }}$. Table III presents some radii of curvature for different steering angles (same for both steering wheels) and distance between axis.

Table III

Curvature radii of rombic vehicle

\begin{tabular}{|c|c|c|}
\hline $\begin{array}{c}\text { Steering } \\
\text { angle }(\theta)\left[{ }^{\circ}\right]\end{array}$ & $\begin{array}{c}\text { Distance } \\
\text { btw. axis }(l)[\mathrm{m}]\end{array}$ & $\begin{array}{c}\text { Curvature } \\
\text { radius }(r)[\mathrm{m}]\end{array}$ \\
\hline 45 & 7 & 4.95 \\
\hline 45 & 6 & 4.24 \\
\hline 30 & 7 & 7.00 \\
\hline 30 & 6 & 6.00 \\
\hline 3.87 & 7 & 37.00 \\
\hline 4.65 & 6 & 37.00 \\
\hline
\end{tabular}

In the sequel, trajectory topology versus kinematic constraints are studied. Graphical simulations of the vehicles were made to show the spanned areas of each type of vehicle. Trajectories refer to a) cart vehicles, b) differential steering vehicles and c) rombic vehicles with the dimensions displayed in Figure 4. The simulated trajectory is 


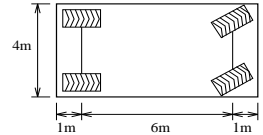

a) Cart

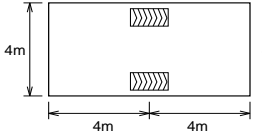

b) Differential drive

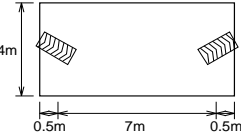

c) Rombic
Fig. 4. Vehicle dimensions used in the simulations.

composed by one line segment followed by two circular arcs with a 5 meters radius and ended with another line segment, (see Figure 5). Tables II and III show that both

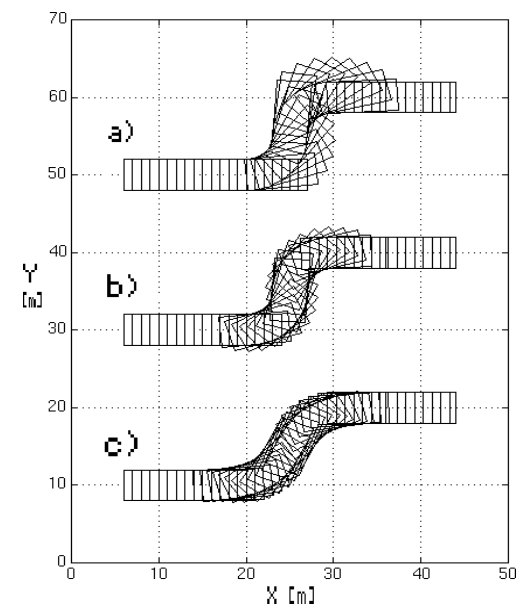

Fig. 5. Trajectory 1. a) Cart, b) Differential steering, c) Rombic.

differential steering and rombic vehicles are capable of executing circular trajectories with a $5 \mathrm{~m}$ radius, thus being able to switch between circular paths in the galleries to radial paths towards docking ports. When trajectory is a line segment, both vehicles span an area with the same width of the vehicles. When following a circular arc with a $5 \mathrm{~m}$ radius, the areas spanned by the vehicles are wider than when following a line segment. For $8 m \times 4 m$ vehicles, and with the geometry displayed in Figure 4, the width of the spanned area when in a $5 \mathrm{~m}$ circular arc is similar: $5.06 \mathrm{~m}$ for differential steering vehicle and $5.28 \mathrm{~m}$ for rombic. Nevertheless, it is also important to quantify the maximum deviation of each vehicle from the designed path. The distances from the center of the spanned areas to the $5 \mathrm{~m}$ radius circular arc are $0.53 \mathrm{~m}$ to the outer side for the differential steering vehicle and $0.79 \mathrm{~m}$ to the inner side for a rombic structure.

Therefore, even though the rombic structure has a slightly larger deviation, this is compensated by the fact that the switching from a circular arc to a line segment is smoother (see Figure 5).

\section{B. Free wheels vs. Air-cushion}

The most usual solution to support the vehicles weight are free wheels. However, a possible alternative for this type of application might be air cushions, considering the range of loads involved. In fact, no industrial application using free wheels was identified for 80 tons loads, while there are solutions which use air cushions. These solu- tions have the advantage of lower contact forces with the corresponding reduction of power requirements for steering and traction motors. Nevertheless, the reduced friction makes the guidance control problem harder, because inertial forces are difficult to handle. Also, a flat and smooth floor and an air compressor are required.

\section{Guidance and Navigation}

This section describes existing solutions for guidance and navigation systems for mobile platforms. The role of these modules on the correct operation of the vehicles is represented in Figure 6.

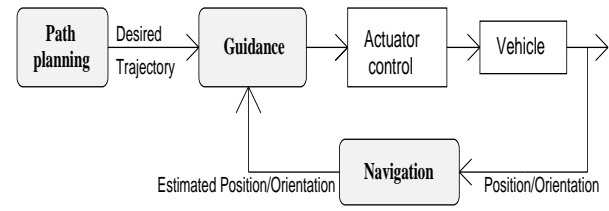

Fig. 6. Block diagram of guidance and navigation systems

General navigation systems determine the current platform's position and orientation on a world frame, based on sensor data. The guidance module compares the vehicle actual location with the desired location along the reference path and dispatches motion commands aiming at minimizing the error. The path planning module establishes the desired trajectory from the initial location to the goal.

\section{A. Guidance}

Guidance, herein understood as the path execution, strongly depends on the type of vehicle kinematics and on the sensors that locate the vehicle. The open possibilities for guidepaths are physical guidepaths, namely optical or inductive, virtual guidepaths and mixed guidepaths. In physical guidepaths the complete net of possible trajectories is specified at floor level. Virtual guidepaths have no physical implementation on the environment being defined at computer level. On the mixed guidepath case, the vehicle will have guidance and navigation capabilities to either follow a physical and a virtual (geometrically defined) path.

On the inductive guidepath strategy, one or more wires are buried on the floor along all the desired trajectories. A low-voltage, low-current, low-frequency, ac signal is conducted through the wire generating a small electricalmagnetic field radiated from the wire. Two joint coils are placed on the vehicle, near the wire. The difference between the induced voltage on both coils is used on a differential control system. This concept leads to very reliable guidance systems being used in industry for years with very good results, [3]. Several vehicles can share the same wire and crossroads can be overcome by the installation of wires with different frequencies to cover all the possible options in a crossroad.

Advantages of the inductive solution include low cost, excellent reliability and the fact that the wire used for steer- 
ing may also provide continuous communication. However, when acting exclusively as an AGV vehicle autonomous maneuvers are limited to a small vicinity of the wire. Therefore, in the case of an AGV robustness (in the sense of ability to recover from path losses) is low. Inductive steering is sensible to external magnetic fields, as opposed to optical inductive steering, [1], where the physical path is defined by a stripe painted on the floor.

When there is no physical path defined on floor level, the guidance module will steer the vehicle along a geometric trajectory defined at the centralized computer based on an optimisation procedure. This procedure takes into account the map of the environment, the transporter actual location, the goal location and the platform's dimensions and kinematics. When this situation arises, it is said that the vehicle performs as a Mobile Robot. During path execution, changes may be made, in real time, to the previously defined geometric trajectory. Those changes may result, for example, from the detection of any unexpected obstacle (i.e., obstacle not represented on the a priori map). Besides this advantage, guidance based on virtual guidepaths are highly flexible for rescue platforms. However, navigation and guidance along a virtual guidepath are more complex than with a physical path, requiring special purpose devices/sensors to be installed. Also, localization errors tend to be larger than for a physical guidepath.

The mixed guidepath concept aims to provide the best of two systems, i.e., accuracy and simplicity along a fixed physical path and flexibility to travel out of the physical path whenever necessary (e.g., to overcome unexpected situations) and to resume again on the physical path some distance away. The particular topic raised for Mixed Vehicles in terms of path planning is the definition of smooth trajectories for leaving and entering into a physical path. As an example, a Mobile Robot may become an AGV near a docking port, but it must first detect and reach the track.

\section{B. Navigation Systems}

Navigation aims at localizing the platform. Its complexity and sensor requirements strongly depends on the type of transportation solution adopted. The location of an AGV is a two-dimensional variable, that describes its pose along the wire. On the contrary, to correctly localize a Mobile Robot, position $(x, y)$ and orientation $(\theta)$ have to be specified, requiring more complex sensors and elaborated signal processing algorithms. The most common methodologies/sensors for continuous localization includes dead reckoning (odometry, inertial navigation) and landmark based procedures, [2].

Dead reckoning is a simple mathematical procedure for determining the present location of a vehicle by using available information about known course and velocity over a given time length. Odometry is the simplest implementation for dead reckoning. Its basic principle is to acquire and process information which is proportional to traveled distance. Usually, odometry localization systems suffer from cumulative errors, thus requiring a periodic error reset based on a more powerful localization system. Combined with other sensors it is possible to build very accurate localization systems. This combined concept, using for example transponders and/or landmark based localization has been used for years in industry with good results.

Landmark navigation is based on the detection of distinct features in the environment (landmarks), whose absolute position is previously known and relative to which a robot can localize itself [2]. Landmarks can be natural or artificial. Natural landmarks are objects that have a function other than robot localization, e.g., doors and walls. Artificial landmarks are specially installed in known places of the environment and are only used for robot localization. They can be either active (e.g., beacons, emitters) or passive (e.g., retroreflector material).

Artificial landmarks have been used in industrial applications for the localization of AGVs and mixed-type vehicles, with very good results. The most commonly used sensor for the detection of the artificial landmarks is a rotating laser emitter/receiver. A horizontal laser beam is emitted and then reflected by landmarks. Based on the received signal, the system can determine the relative orientation between the landmark and the vehicle. Position and orientation of the vehicle on a global frame are then computed by a coordinate transformation using a landmark map (landmark absolute positions) that has to be kept in memory. This localization procedure is robust to magnetic fields, and has an easy installation. Besides, landmarks are passive and inexpensive and laser is very accurate (the accuracy of the state of the art laser systems can go up to $2-3 \mathrm{~mm}$ ). However, laser systems are expensive, may require complex computations and must usually be combined with odometry to ensure absolute positioning.

\section{CONCLUSIONS}

Should a flexible guidance transportation solution be adopted for ITER RH system, it is proposed to use fixed guidepaths whenever possible and virtual guidepaths in special situations. This need may arise if a vehicle has a malfunction, stops and occupies the fixed guidepath. The flexibility provided by a mixed guidepath methodology is also extremely important for the rescue platforms. When approaching docking ports a fixed guidepath can be of extreme use once it allows very precise docking of the vehicle, [1]. However, navigation and guidance of mixed-type vehicles is more complex, since it requires the coordination of a larger number of sensors and of the switching between operation modes.

\section{REFERENCES}

[1] M.I.Ribeiro, P.Lima, P.Aparício, R.Ferreira, "Conceptual Study on Flexible Guidance, Navigation and Docking Systems for ITER RH Transport Casks," Techn.Rep. ISR-403-97, Instituto de Sistemas e Robótica, Instituto Superior Técnico, April 1997.

[2] J.Borenstein,H.R.Everett,L.Feng, "Where am I? - Sensors and Methods for Mobile Robot Positioning," The University of Michigan, 1996.

[3] Gary Hammond, "AGVs at Work - Automated Guided Vehicles Systems," IFS(publications) Ltd, UK - Springer Verlag, 1986. 